\title{
Amelioration of ischemic brain damage by peritoneal dialysis
}

\author{
María del Carmen Godino, ${ }^{1}$ Victor G. Romera, ${ }^{2}$ José Antonio Sánchez-Tomero, ${ }^{3}$ \\ Jesus Pacheco, ${ }^{4}$ Santiago Canals, ${ }^{4}$ Juan Lerma, ${ }^{4}$ José Vivancos, ${ }^{5}$ María Angeles Moro, ${ }^{2}$ \\ Magdalena Torres, ${ }^{1}$ Ignacio Lizasoain, ${ }^{2}$ and José Sánchez-Prieto ${ }^{1}$
}

\begin{abstract}
1Departamento de Bioquímica y Biología Molecular, Facultad de Veterinaria, Universidad Complutense, Madrid, Spain. ²Departamento de Farmacología, Facultad de Medicina, Universidad Complutense, Madrid, Spain. ${ }^{3}$ Servicio de Nefrología, Hospital Universitario de la Princesa, Madrid, Spain. ${ }^{4}$ Instituto de Neurociencias de Alicante, Universidad Miguel Hernández-Consejo Superior de Investigaciones Científicas, Sant Joan d'Alacant, Spain. ${ }^{5}$ Servicio de Neurología, Hospital Universitario de la Princesa, Madrid, Spain.
\end{abstract}

\begin{abstract}
Ischemic stroke is a devastating condition, for which there is still no effective therapy. Acute ischemic stroke is associated with high concentrations of glutamate in the blood and interstitial brain fluid. The inability of the tissue to retain glutamate within the cells of the brain ultimately provokes neuronal death. Increased concentrations of interstitial glutamate exert further excitotoxic effects on healthy tissue surrounding the infarct zone. We developed a strategy based on peritoneal dialysis to reduce blood glutamate levels, thereby accelerating brain-to-blood glutamate clearance. In a rat model of stroke, this simple procedure reduced the transient increase in glutamate, consequently decreasing the size of the infarct area. Functional magnetic resonance imaging demonstrated that the rescued brain tissue remained functional. Moreover, in patients with kidney failure, peritoneal dialysis significantly decreased glutamate concentrations. Our results suggest that peritoneal dialysis may represent a simple and effective intervention for human stroke patients.
\end{abstract}

\section{Introduction}

Stroke is one of the leading causes of death and disability worldwide, for which no effective neuroprotective therapy exists. Ischemic brain damage is triggered by excessive release of the excitatory neurotransmitter L-glutamate $(1,2)$ as a result of energy failure and ion gradient collapse, resulting in a reversal of glutamate uptake via glutamate transporters $(3,4)$. Excessive glutamate-evoked $\mathrm{Ca}^{2+}$ entry via NMDA receptors further promotes cell death by triggering an excitotoxic cascade that involves the activation of $\mathrm{Ca}^{2+}$-dependent enzymes, the disruption of mitochondrial function, and cell necrosis or apoptosis (5). Despite intense research efforts, suitable pharmacological strategies to enhance neuroprotection of ischemic tissues remain elusive (6), partly because pharmacotherapy tends to target a single step of the complex excitotoxic cascade and it does not distinguish between damaged and healthy tissue.

After acute ischemic stroke, there is an increase in glutamate levels in the blood (7), most likely due to enhanced brain-to-blood efflux $(8,9)$ that is driven by increased interstitial glutamate concentrations (10). We reasoned that peritoneal dialysis could decrease the blood levels of glutamate, thereby minimizing the interstitial glutamate in the brain and curtailing ischemia-induced brain damage $(8,9)$.

\section{Results and Discussion}

We investigated the hypothesis that peritoneal dialysis could decrease the blood levels of glutamate, thereby minimizing brain damage in a model of brain ischemia in which rats were subjected to permanent middle cerebral artery occlusion (pMCAO) (Figure 1A). The concentration of glutamate transiently increased in plasma 4.5 and 5.5 hours after ischemia (pMCAO; Figure 1B),

Authorship note: María del Carmen Godino and Victor G. Romera contributed equally to this work.

Conflict of interest: The authors have declared that no conflict of interest exists. Citation for this article: J Clin Invest. 2013;123(10):4359-4363. doi:10.1172/JCI67284. and a corresponding cerebral infarct of $23.3 \% \pm 1.3 \%(n=9)$ was observed 24 hours after pMCAO (Figure 1, D and E). Peritoneal dialysis is a procedure used to treat patients with severe chronic kidney disease, whereby fluids and dissolved substances are exchanged between the blood and the dialysate across the peritoneum $(11,12)$. In rats subjected to PMCAO, peritoneal dialysis 2.5 hours after PMCAO significantly attenuated the increase in plasma glutamate induced by ischemia (pMCAO plus dialysis at 2.5 hours; Figure 1B), and, importantly, this decrease in plasma glutamate levels was associated with a significant reduction in the volume of cerebral infarct $(12.1 \% \pm 2.2 \%, n=5, P<0.001)$ (Figure 1, D and E). Confirming that rat peritoneal dialysis leads to a reduction of plasma glutamate, we found that the accumulated glutamate in the dialysate after 1 hour of dialysis was $59.2 \pm 12.2 \mu \mathrm{M}(n=8)$. As a control, we added glutamate to the dialysate infusion to cancel the concentration gradient for glutamate, therefore preventing its clearance from the blood. Indeed, the addition of glutamate $(400 \mu \mathrm{M})$ to the dialysate abolished the glutamate clearance observed following peritoneal dialysis, resulting in a significant increase in blood glutamate concentration after pMCAO (pMCAO plus dialysis at 2.5 hours plus $400 \mu \mathrm{M}$ glutamate; Figure 1C) and, importantly, abrogated the beneficial effect of peritoneal dialysis on cerebral infarct size (Figure 1D). In sham-operated rats, in which middle cerebral arteries were exposed but not occluded, no changes in plasma glutamate concentration were detected (Figure 1C) and no cerebral infarct was observed (Figure 1D). The changes in plasma glutamate correlated well with the size of cerebral infarct measured 24 hours after insult $\left(r^{2}=0.5312\right.$, $P=0.0021$; Figure 1E). We also observed that peritoneal dialysis is equally efficient in reducing the infarct volume when starting 5 hours after $\mathrm{pMCAO}(15.0 \% \pm 1.2 \%, n=7, P<0.01$; Figure 1 , A and D $)$, as plasma glutamate at 5.5 hours after pMCAO is still high and close to maximal levels (Figure 1B). These data indicate that by decreasing the glutamate concentration in the blood, peritoneal 
A
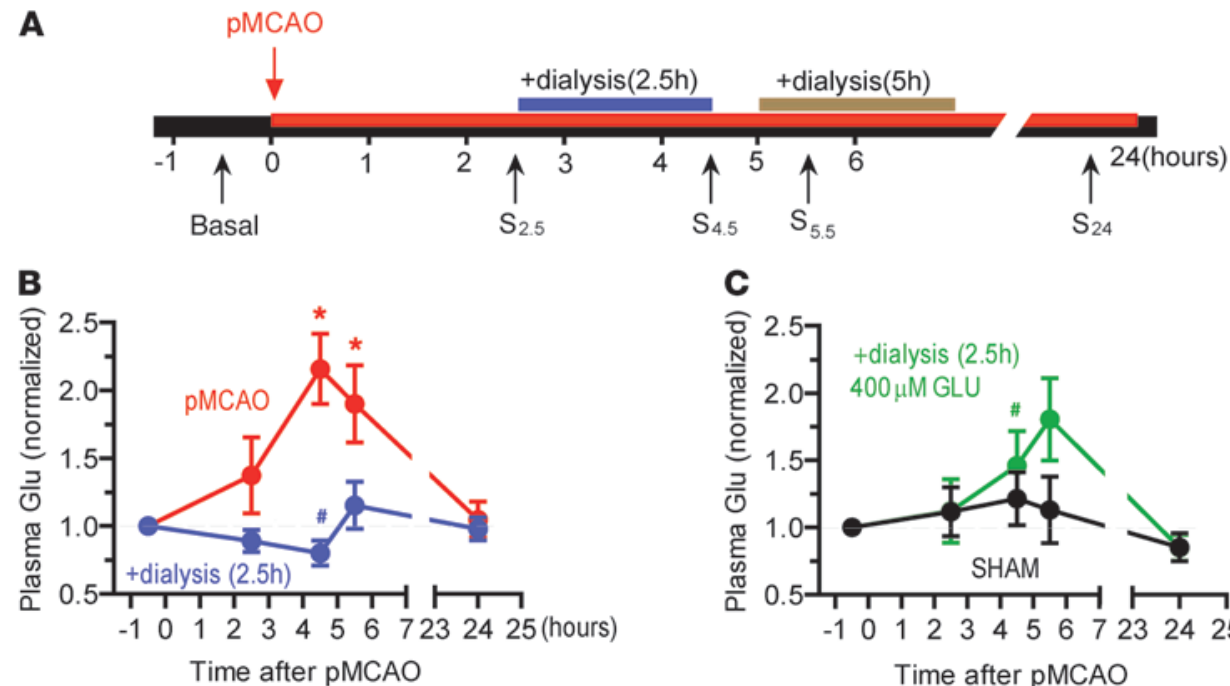

C
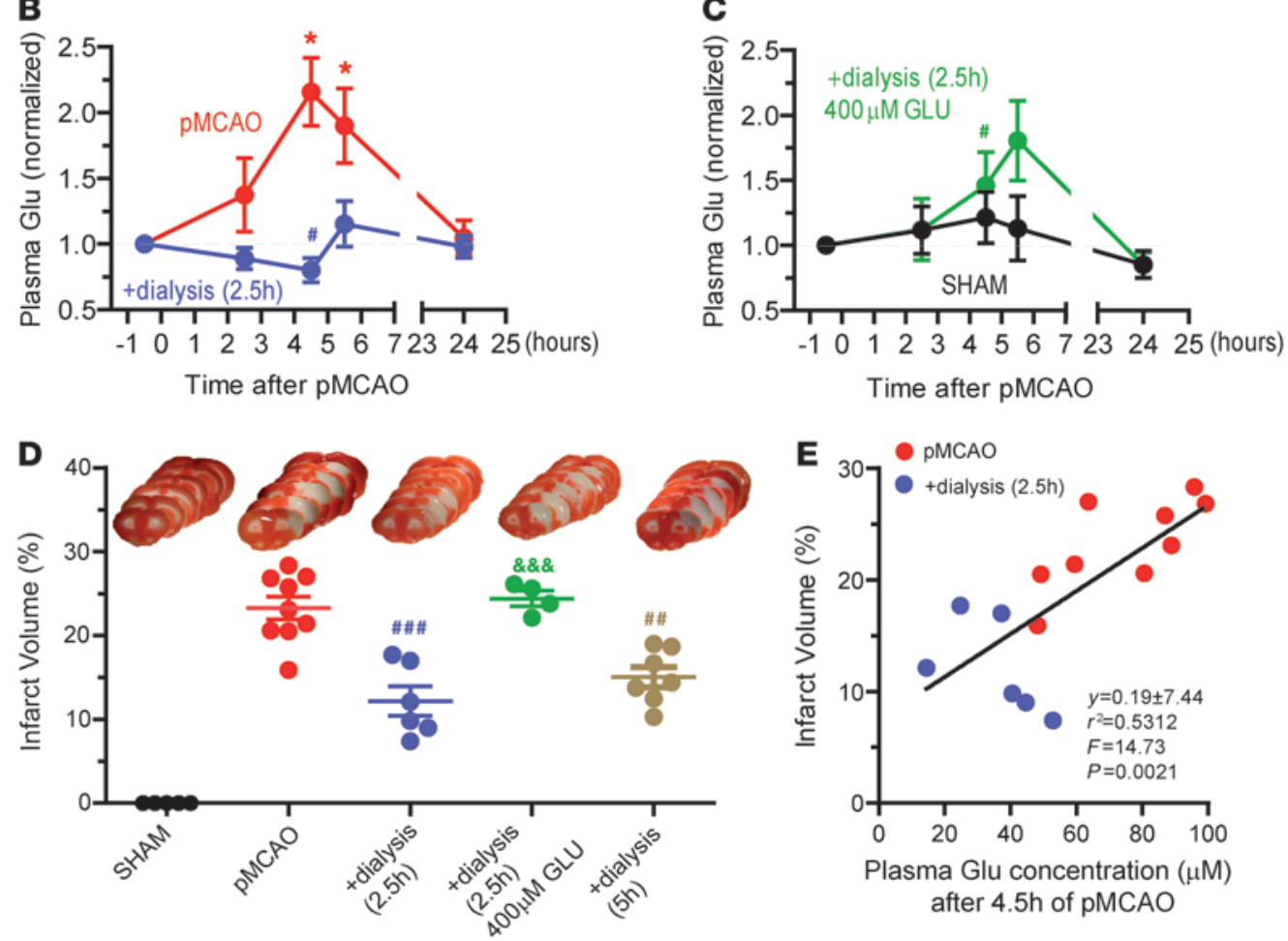

\section{Figure 1}

Peritoneal dialysis attenuates ischemia-induced increases in plasma glutamate concentration and cerebral infarct size. (A) The glutamate concentration was determined in plasma samples taken prior to (basal) and at different times (hours) after pMCAO (samples were taken at 2.5 hours [S2.5], 4.5 hours [S4.5], 5.5 hours [S5.5], and 24 hours [S24]), with peritoneal dialysis initiated 2.5 hours after pMCAO induction. Cerebral infarct size was measured 24 hours after pMCAO. Plasma glutamate (normalized to basal levels) and cerebral infarct size were determined in the following conditions: (B, D, and E) pMCAO as well as pMCAO plus peritoneal dialysis at 2.5 hours [+dialysis (2.5h)]; (C and $\mathbf{D})$ pMCAO plus peritoneal dialysis at 2.5 hours with $400 \mu \mathrm{M}$ glutamate in the dialysate [+dialysis (2.5h) $400 \mu \mathrm{M}$ GLU] as well as sham-operated rats (SHAM); and (D) pMCAO plus peritoneal dialysis at 5 hours [+dialysis (5h)]. (E) Correlation between plasma glutamate (measured 4.5 hours after pMCAO) and infarct size. The average basal glutamate values were $51.8 \pm 2.6 \mu \mathrm{M}(n=21)$. Data represent the mean $\pm \mathrm{SEM}$, and $n=5-9$ for each condition. ${ }^{*} P<0.05$ versus basal values (ANOVA); ${ }^{*} P<0.05, \# \#<0.01$, \#\#\# $P<0.001$ versus corresponding values in $\mathrm{pMCAO}$ (ANOVA); \&\&\& $P<0.001$ versus corresponding values in [+dialysis (2.5h)] (ANOVA).

dialysis effectively promotes brain-to-blood glutamate efflux (9), minimizing the ischemic increase in extracellular glutamate and the resulting tissue damage.

Despite the apparent beneficial effects of peritoneal dialysis, it remains unclear whether the tissue rescued from death, according to structural and anatomical parameters, remains functional. To clarify this issue, we used blood oxygenation level-dependent (BOLD) functional magnetic resonance imaging (fMRI) to determine tissue functionality (13). AMRI experiments were performed 14 days after the ischemic insult in treated (dialyzed) and untreated animals. Electric stimulation of the corpus callosum produces widespread $\mathrm{AMRI}$ activation maps in the cerebral cortex (Figure 2A). The time course of BOLD signals during callosal activation showed fully recovered hemodynamic responses after every stimulation epoch and the absence of movement artifacts or spontaneous poststimulus activations suggestive of seizures (Figure 2E). Functional activation extends to both hemispheres almost symmetrically in intact animals (Figure 2A). The symmetry was lost in pMCAO animals due to the ischemic injury (Figure 2A). To quantify tissue viability, we defined a functionality index as the ratio of cortical volume activated in the infarcted hemisphere divided by the contralateral (spared) side, during callosal stimulation. Intact animals were used as reference. Thus, the functionality index measures the extension of the functional lesion normalized to the healthy hemisphere and decreases experimental variability. As shown in Figure 2B, pMCAO induced a severe loss of cortical viability. Peritoneal glutamate dialysis partially prevented this functional damage, rescuing tissue viability (Figure 2B). In the same animals, we also measured the infarct size based on the volume of the T2-hyperintense signal in MRI anatomical scans and 
A
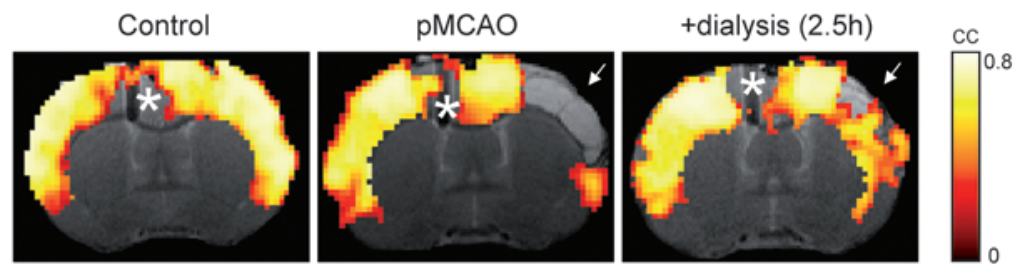

B

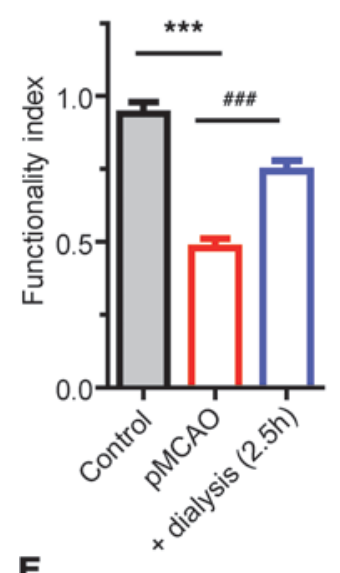

C

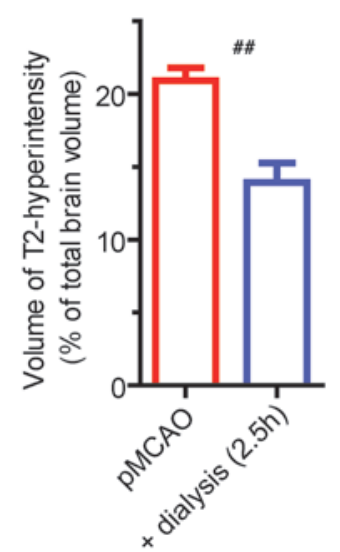

E

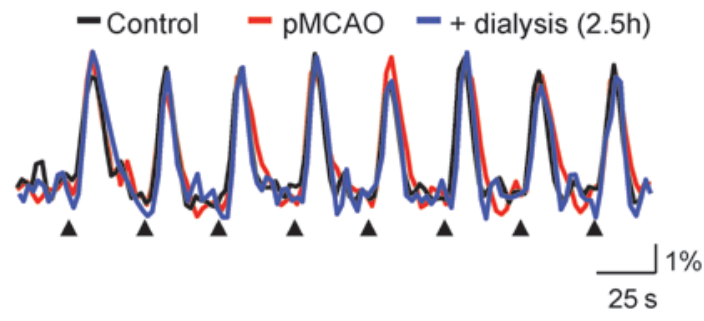

D

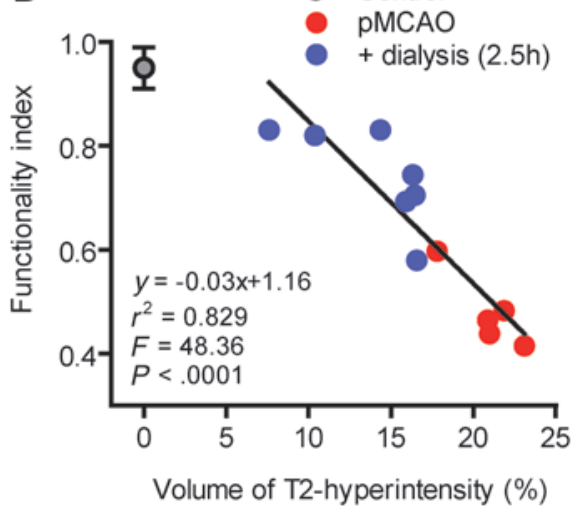

F

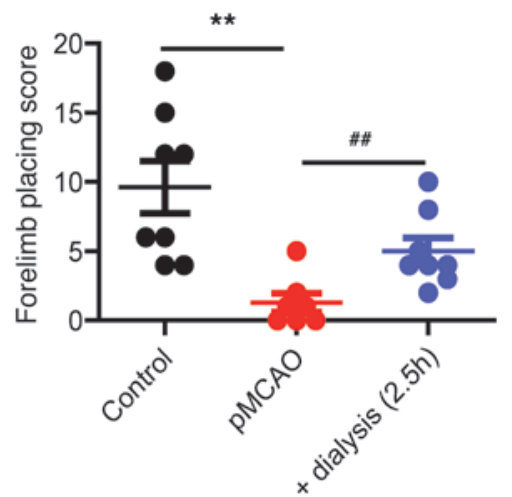

Figure 2

Functional recovery of the cerebral cortex.(A) Representative cortical activation during corpus callosum stimulation. fMRI maps are thresholded at $P<0.0001$ (uncorrected) and overlaid on anatomical scans. Electrode artifact is marked with asterisks, and the arrows point toward the infarct area. Color-coded scale represents correlation of the BOLD signal with the stimulation paradigm. Note almost symmetric bilateral activation of the cortex in healthy animals upon corpus callosum stimulation. (B) Functionality index measured as the volume of active tissue in the right (ischemic) cortex normalized by the left (healthy) cortex. Intact animals (black, $n=3$ ) are used as control for animals subjected to pMCAO (red, $n=5$ ) and PMCAO plus peritoneal dialysis at 2.5 hours (blue, $n=7$ ). Signal loss introduced by the implanted electrode (asterisk in A) was corrected. Data represent mean \pm SEM. (C) Volume of the hyperintense T2-weighted signal (arrows in A). Data represent mean \pm SEM. (D) Highly significant negative correlation was found between the volume of the ischemic lesion (measured in $\mathbf{C}$ ) and the functionality index (measured in B). (E) Average hemodynamic signal in response to callosal stimulation across all significative voxels in $\mathbf{A}(P<0.0001$, uncorrected). Stimulation epochs are marked by arrowheads. (F) Behavioral testing of the simultaneous use of both forelimbs on cylinder wall during exploratory activity. Data represent the mean \pm SEM $(n=5-7)$. Statistical analysis was performed by (B) ANOVA followed by Bonferroni's test, $(\mathbf{C}$ and $\mathbf{F})$ 2-tailed Student's $t$ test, or (D) linear regression. ${ }^{* \star} P<0.01,{ }^{\star * \star} P<0.001$ control versus $\mathrm{pMCAO} ;{ }^{\# \#} P<0.01$, ${ }^{\# \# \#} P<0.001$ pMCAO versus + dialysis (2.5h).

were able to demonstrate not only the reduced lesion volume under dialysis (Figure 2C), but also a tight correlation between the volume of the T2 lesion and the calculated functionality index (Figure 2D). As a whole, these findings demonstrate that the reduction in infarct volume by dialysis preserves neuronal integrity and confirms the validity of anatomical measures of ischemic lesion based on T2-weighted images as predictors of functionality. In good agreement with the fMRI results, we were able to demonstrate that the functionality of the system, measured behaviorally using the limb-use asymmetry test, was also largely preserved in dialyzed animals (Figure 2F). Taken together, our results indicate that the functional deficit produced by ischemic insult can be partially prevented by peritoneal dialysis.

The endothelial cells that form the blood-brain barrier are endowed with facilitative and $\mathrm{Na}^{+}$-dependent glutamate transporters in the luminal and abluminal membranes, respectively, that mediate glutamate efflux to the blood. Glutamate is actively transported from the interstitial space to the endothelial cells and 


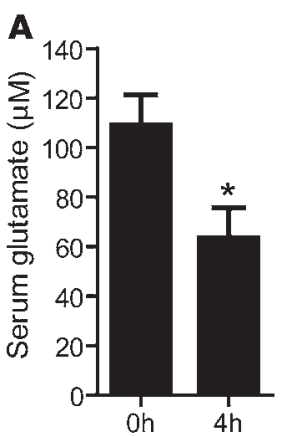

Time in dialysis

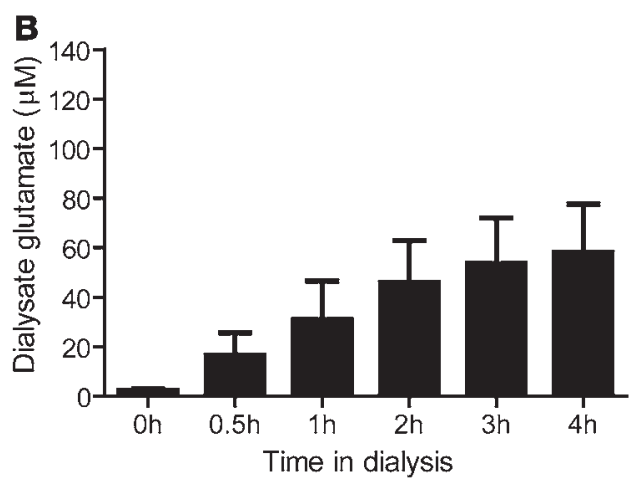

\section{Figure 3}

Peritoneal dialysis decreases serum glutamate levels in patients with severe chronic kidney disease. (A) Serum glutamate concentration in patients prior to and after one 4-hour session of peritoneal dialysis. Glutamate levels were determined fluorimetrically using glutamate dehydrogenase. The serum glutamate concentrations were $109.2 \pm 17.6 \mu \mathrm{M}$ and $63.6 \pm 10.7 \mu \mathrm{M}$ before and after a 4-hour dialysis session, respectively. (B) Time-dependent accumulation of glutamate in the dialysate. The results represent the mean \pm SEM $(n=7) .{ }^{*} P<0.05$ as compared with serum glutamate concentration prior to the dialysis session (Student's $t$ test). from the endothelial cells into the circulation via facilitative transporters (8). Peritoneal dialysis is primarily used to treat patients with chronic kidney disease (14), which constitute approximately $15 \%$ of all dialysis patients. Thus, to further demonstrate the validity of our proposal that peritoneal dialysis promotes brain-toblood glutamate efflux, we analyzed serum glutamate concentrations in patients before and after dialysis. One session of peritoneal dialysis significantly reduced serum glutamate levels in patients with severe chronic kidney disease (Figure 3A). As expected, glutamate rapidly accumulated in the dialysate $(58.0 \pm 19.5 \mu \mathrm{M}$ after 4 hours of dialysis; Figure 3B), almost reaching serum glutamate concentrations, indicating that glutamate is rapidly exchanged from the blood to the dialysate. These results demonstrate that the peritoneum promotes rapid and efficient glutamate exchange between the blood and the dialysate and that peritoneal dialysis induces rapid clearance of interstitial glutamate. Based on these findings, we propose that peritoneal dialysis may offer a suitable treatment to decrease blood glutamate concentrations in acute stroke patients, efficiently reducing interstitial glutamate levels and ultimately ameliorating cerebral damage.

Synaptically released glutamate is nontoxic to neurons because its diffusion, binding, and transport via $\mathrm{Na}^{+}$-dependent glutamate transporters rapidly lower the concentration of this glutamate to concentrations in the submicromolar range, preventing tonic activation of NMDA receptors $(15,16)$. By contrast, cerebral ischemia provokes a more sustained increase in extracellular glutamate concentration (10) that can exert toxic effects on metabolically viable tissue around the infarct area $(17,18)$. Indeed, plasma glutamate concentrations remain elevated in patients with progressing ischemic stroke 24 hours after stroke (7), reflecting the ongoing cell death. Moreover, polymorphisms affecting glutamate uptake are associated with elevated levels of plasma glutamate and a poorer neurological outcome following stroke (19). Therapeutic interventions based on impeding glutamate receptor activation have so far failed to ameliorate tissue degeneration after stroke. However, the present findings suggest that removing glutamate from the extracellular space may represent an effective means of preventing tissue damage surrounding the infarct area. Interestingly, decreases in serum glutamate levels induced by intravenous injection of oxalacetate and pyruvate $(9,20)$ also produce neuroprotection in both focal (21) and global brain ischemia $(22,23)$ and in traumatic brain injury $(24)$. However, undesirable side effects of this approach have been reported in vivo, including enhanced excitatory transmission in the cortex (25).

Peritoneal dialysis overcomes some of the drawbacks associated with the pharmacological neuroprotectants that have failed to produce beneficial effects in clinical trials. First, rather than targeting a single step of the complex excitotoxic cascade initiated by glutamate $(5,17,26)$, peritoneal dialysis removes the source of the problem, the interstitial glutamate itself, thereby preventing excitotoxicity around the damaged tissue. Second, neuroprotective drugs do not distinguish between damaged and healthy tissue, and, thus, NMDA antagonists will block NMDA receptor-driven $\mathrm{Ca}^{2+}$ influx in the infarct area (27) while also altering NMDA receptor-dependent synaptic plasticity in healthy brain regions (28). By contrast, the peritoneal dialysis procedure removes interstitial glutamate preferentially from the infarct areas that contain the higher brain/blood glutamate gradients. Furthermore, the use of peritoneal dialysis may substantially reduce the time of treatment.

Most strokes are ischemic and occur after vessel occlusion, while about $15 \%$ are hemorrhagic in nature. Thrombolysis with recombinant tissue plasminogen activator (rtPA) is the only treatment approved for reperfusion and reversal of vessel obstruction, although in practice this treatment is available to a limited number of patients (6). Administration of rtPA requires prior distinction between thrombotic and hemorrhagic stroke, as this treatment is not suitable for hemorrhagic stroke patients. Accordingly, peritoneal dialysis has the additional advantage that it is applicable in cases of both ischemic and hemorrhagic stroke, as the elevation of interstitial glutamate is a feature common to both conditions (29). Other conditions characterized by increases in brain glutamate levels, such as glioma-induced seizures (30), may also benefit from this approach. Moreover, peritoneal dialysis can be implemented rapidly by the nephrologist when the patient arrives to hospital. The short time required for catheter implantation (15 minutes) ensures that peritoneal dialysis can be applied well within the time window for glutamate clearance and for therapeutic effect. Finally, the use of peritoneal dialysis for the treatment of stroke would benefit greatly from the widespread experience already attained by medical personnel in the use of this technique. Nonetheless, preclinical work is needed in order to prove that peritoneal dialysis is also effective and safe in aged and/or comorbid animals, reflecting the clinical situation, and clinical trials are required to demonstrate the efficacy of peritoneal dialysis in acute ischemic stroke and other brain pathologies.

\section{Methods}

Further information is available in the Supplemental Methods (supplemental material available online with this article; doi:10.1172/JCI67284DS1).

PMCAO. The PMCAO was achieved by ligature as described previously (31). In sham-operated control rats, the middle cerebral artery was exposed but not occluded. 
Statistics. Data are expressed as the mean \pm SEM. ANOVA with Tukey's multiple comparison test, ANOVA followed by Bonferroni's test, or 2 -tailed Student's $t$ test were used. A $P$ value of less than 0.05 was considered significant.

Study approval. All patients provided informed consent prior to participation in this study. All the experimental procedures were performed in accordance with the guidelines and with the approval of the Animal Welfare Committee of the Universidad Complutense (DC 86/609/EU).

\section{Acknowledgments}

This work was supported by grants from the Spanish MINECO to J. Sánchez-Prieto (BFU2010/16947), I. Lizasoain (SAF201123354), and M.A. Moro (SAF2009-08145, SAF2012-33216, and CSD2010-00045); from Fondo Europeo de Desarrollo Regional (FEDER) “Instituto de Salud Carlos III” (RD06/0026, RD12/0014) to I. Lizasoain, M. Torres, J. Vivancos, and J. Sánchez-Prieto; from the "Comunidad de Madrid" (CAM-I2M2 2011-BMD-2349) to I.
Lizasoain, M. Torres, J. Vivancos, and J. Sánchez-Prieto; and from NEUROSTEMCM to M.A. Moro (S2010/BMD-2336). Research in the laboratory of S. Canals and J. Lerma is supported by grants from the Spanish MINECO (BFU2009-09938, BFU2011-24084, and CSD2007-00023). We thank M. Sefton for editorial assistance and Begoña Fernández for excellent technical assistance.

Received for publication October 11, 2012, and accepted in revised form July 3, 2013.

Address correspondence to: Ignacio Lizasoain, Departamento de Farmacología, Facultad de Medicina, Universidad Complutense, Madrid-28040, Spain. Phone: 34.91.3941465; Fax: 34.91.3941464; E-mail: ignacio.lizasoain@med.ucm.es. Or to: José Sánchez-Prieto, Departamento de Bioquímica y Biología Molecular, Facultad de Veterinaria, Universidad Complutense, Madrid-28040, Spain. Phone: 34.91.3943891; Fax: 34.91.3943909; E-mail: jsprieto@vet.ucm.es.
1. Choi DW, Rothman SM. The role of glutamate neurotoxicity in hypoxic-ischemic neuronal death. Annu Rev Neurosci. 1990;13:171-182.

2. Lipton P. Ischemic cell death in brain neurons. Physiol Rev. 1990;79(4):1431-1568.

3. Sánchez-Prieto J, González MP. Occurrence of a large $\mathrm{Ca}^{2+}$-independent release of glutamate during anoxia in isolated nerve terminals. J Neurochem. 1988;59(4):1322-1325.

4. Rossi D, Oshima T, Attwell D. Glutamate release in severe brain ischaemia is mainly by reversed uptake. Nature. 2000;403(6767):316-321.

5. Moskowitz MA, Lo EH, Iadecola C. The science of stroke: mechanisms in search of treatments. Neuron. 2010;67(2):181-198.

6. Endres $M$, et al. Improving outcome after stroke: overcoming the translational roadblock. Cerebrovasc Dis. 2008;25(3):268-278.

7. Castillo J, Dávalos A, Noya M. Progression of ischaemic stroke and excitotoxic aminoacids. Lancet. 1997;349(9045):79-83.

8. Hawkins RA, O'Kane R, Simpson IA, Viña JR. Structure of the blood-brain barrier and its role in the transport of amino acids. J Nutr. 2006; 136(suppl):218S-226S.

9. Teichberg VI, Cohen-Kashi-Malina K, Cooper I, Zlotnik A. Homeostasis of glutamate in brain fluids: an accelerated brain-to-blood efflux of excess glutamate is produced by blood glutamate scavenging and offers protection from neuropathologies. Neuroscience. 2009;158(1):301-308.

10. Benveniste H, Drejer J, Schousboe A, Diemer NH. Elevation of the extracellular concentrations of glutamate and aspartate in rat hippocampus during transient cerebral ischemia. J Neurochem.
1984;43(5):1369-1374.

11. Gokal R. Peritoneal dialysis in the 21st century: an analysis of current problems and future developments. J Am Soc Nephrol Suppl. 2002; 13(5):S104-S116.

12. Johansson L, Brown EA. Peritoneal dialysis. Semin Dial. 2009;22(1):27-29.

13. Logothetis NK. What we can do and what we cannot do with fMRI. Nature. 2008; 453(7197):869-878.

14. Jain N, Simoyi P. An overview of chronic kidney disease management and CAPD in the home. $\mathrm{BrJ}$ Community Nurs. 2008;13(5):213-218.

15. Bergles DE, Jahr CE. Synaptic activation of glutamate transporters in hippocampal astrocytes. Newron. 1997;19(6):1297-1308

16. Attwell D, Gibb A. Neuroenergetics and the kinetic design of excitatory synapses. Nat Rev Neurosci. 2005;6(11):841-849.

17. Dirnagl U, Iadecola C, Moskowitz MA. Pathobiology of ischemic stroke: an integrated view. Trends Neurosci. 1999;22(9):391-397.

18. Dreier JP. The role of spreading depression, spreading depolarization and spreading ischemia in neurological disease. Nat Med. 2011;17(4):439-447.

19. Mallolas J, et al. A polymorphism in the EEAT2 promoter is associated to higher glutamate concentrations an higher frequency of progressing stroke. J Exp Med. 2006;203(3):711-717.

20. Gottlieb M, Wang Y, Teichberg VI. Blood-mediated scavenging of cerebrospinal fluid glutamate. J Neurochem. 2003;87(1):119-126.

21. Nagy D, et al. Oxalacetate decreases the infarct size and attenuates the reduction in evoked responses after photothrombotic focal ischemia in the rat cortex. Cell Mol Neurobiol. 2009;29(6-7):827-835.

22. Marosi M, et al. Oxalacetate restores the long-term potentiation impaired in CA1 region by 2 -vessel occlusion. Eur J Pharmacol. 2009;604(1-4):51-57.

23. Campos F, et al. Neuroprotection by glutamate oxalacetate transaminase in ischemic stroke: an experimental study. J Cereb Blood Flow Metab. 2011;31(6):1378-1386

24. Zlotnik A, et al. The contribution of the blood glutamate scavenging activity of pyruvate to its neuroprotective properties in a rat model of close head injury. Neurochem Res. 2008;33(6):1044-1050.

25. Nagy D, et al. Effects of blood glutamate scavenging on cortical evoked potentials. Cell Mol Neurobiol. 2010;30(7):1101-1106.

26. Choi DW, Maulucci-Gedde M, Kriegstein AR. Glutamate neurotoxicity in cortical cell culture. J Nenrosci. 1987;7(2):357-368.

27. Albers GW, Goldberg MP, Choi DW. N-methyl-D-aspartate antagonists: ready for clinical trials in brain ischemia. Ann Neurol. 1989;25(4):398-403.

28. Malenka RC, Nicoll RA. NMDA-receptor-dependent synaptic plasticity: multiple forms and mechanisms. Trends Neurosci. 1993;16(12):521-527.

29. Qureshi AI, et al. Extracellular glutamate and other amino acids in experimental intracerebral hemorrhage: an in vivo microdialysis study. Crit Care Med. 2003;31(5):1482-1489.

30. Buckingham SC, et al. Glutamate release by primary brain tumors induces epileptic activity. Nat Med. 2011;17(10):1269-1274.

31. Sobrado $\mathrm{M}$, et al. Longitudinal studies of ischemic penumbra by using 18F-FDG PET and MRI techniques in permanent and transient focal cerebral ischemia in rats. Neuroimage. 2011;57(1):45-54. 